\title{
IceCube-Gen2: the next-generation neutrino observatory for the South Pole
}

\author{
The IceCube-Gen2 Collaboration ${ }^{\dagger}$ \\ ${ }^{\dagger}$ http://icecube.wisc.edu/collaboration/authors/icrc17_gen2 \\ E-mail: jvansanten@icecube.wisc.edu
}

\begin{abstract}
The IceCube Neutrino Observatory is a cubic-kilometer Cherenkov telescope buried in the ice sheet at the South Pole that detects neutrinos of all flavors with energies from tens of GeV to several PeV. The instrument provided the first measurement of the flux of high-energy astrophysical neutrinos, opening a new window to the non-thermal universe. Here we present design studies for IceCube-Gen2, the next-generation neutrino observatory for the South Pole. IceCube-Gen2 will have an instrumented volume nearly 10 times greater than IceCube alone, substantially increasing sensitivity to high-energy neutrinos. On the surface, a large air shower detector will veto high-energy atmospheric muons and neutrinos from the southern hemisphere, enhancing the reach of astrophysical neutrino searches. In the ice, a number of new optical module designs currently being evaluated will allow for substantially increased photosensitive area per unit cost. We show how these different sensor designs affect the instrument's ability to resolve the sources of astrophysical neutrinos.
\end{abstract}

Corresponding author: J. van Santen*

DESY, Platanenallee 6, D-15738 Zeuthen, Germany

35th International Cosmic Ray Conference - ICRC2017

10-20 July, 2017

Bexco, Busan, Korea

${ }^{*}$ Speaker. 


\section{From IceCube to IceCube-Gen2}

IceCube is a cubic-kilometer Cherenkov telescope buried in the ice sheet at the South Pole [1] that detects neutrinos of all flavors with energies from tens of GeV to several PeV. While the instrument provided the discovery of the flux of high-energy astrophysical neutrinos $[2,3,4,5]$, the sources of these neutrinos have remained out of reach [6]. Learning more about the sources and resolving their locations will require collecting significantly more neutrinos per year than are possible with IceCube alone. To that end, design studies are underway for IceCube-Gen2, a nextgeneration neutrino observatory for the South Pole.

IceCube-Gen2 will encompass the existing IceCube array [1], covering the $\mathrm{TeV}$ range, as well as an array of new, widely-spaced strings to increase sensitivity above $\sim 10 \mathrm{TeV}$. Additional components would expand IceCube-Gen2 into a wide-band neutrino facility. A large surface array would lower the energy threshold for southern-sky neutrino searches by vetoing mildly-inclined air showers [8, 9]. A radio array would detect cosmogenic neutrinos above $\sim 100 \mathrm{PeV}[10]$. A dense infill inside the existing DeepCore would extend atmospheric oscillation measurements and dark matter searches down to several GeV [11]. Here we focus on the sensitivity characterization of the high-energy array and the surface veto array.

The high-energy array will detect neutrinos primarily via two channels: tracks, produced by relativistic muons crossing the detector, and cascades, isolated energy depositions induced by charged-current $v_{e}, v_{\tau}$, and neutral-current interactions of all neutrino flavors. Sensitivity to individual sources scales with the rate of track events, and thus with the projected area of the detector, while sensitivity to the energy spectrum and flavor composition of the high-energy diffuse flux scales with the rate of cascade events, and thus the instrumented volume. Several geometries are under consideration for the high-energy array, with instrumented volumes ranging from 6.2 to $9.5 \mathrm{~km}^{3}$. Each detector geometry strikes a different compromise between projected area and contained volume. For the studies described here we focus on the "sunflower" layout shown in Fig. 1.

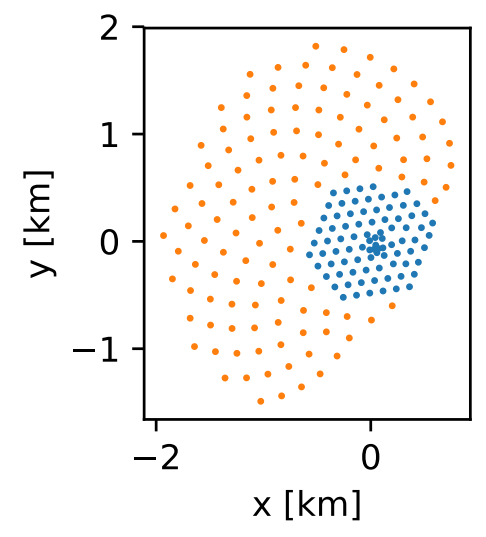

Figure 1: View of IceCube-Gen2 strings from above in the "sunflower" layout. The 120 new strings (shown as orange points) are spaced $240 \mathrm{~m}$ apart and instrumented with 80 optical modules over a vertical length of $1.25 \mathrm{~km}$. The total instrumented volume is $7.9 \mathrm{~km}^{3}$, nearly 10 times larger than IceCube alone (blue points). 


\section{Sensitivity to astrophysical neutrinos}

We estimate the sensitivity of each proposed detector configuration to various astrophysical neutrino production scenarios using a set of mock likelihood analyses modeled on previous IceCube results. In the following we present our method for obtaining neutrino event rates from basic detector performance quantities such as muon effective area and energy resolution. We then briefly discuss the likelihood technique. Finally, we use the technique to demonstrate the potential of the IceCube-Gen2 high-energy array to measure properties of the observed quasi-diffuse neutrino flux and discover steady point sources of neutrinos.

\subsection{Event rate calculation}

We have parameterized the performance of each of our proposed detector geometries to detect and reconstruct tracks and cascades. We then calculate event rates by folding these quantities with the probabilities for neutrinos of a particular flavor and energy to produce one of these two event signatures. This approach allows us to separate the performance of the detector from limitations imposed by the physics of neutrino interactions, e.g. absorption in the Earth.

For muon tracks, the detector performance is characterized by four quantities: muon effective area, surface veto coverage, energy resolution, and point spread function.

Muon effective area The left panel of Fig. 2 shows the muon effective area of the $240 \mathrm{~m}$ "sunflower" geometry. For PeV muons it is nearly identical to the instrumented area, but drops significantly below $10 \mathrm{TeV}$ due to large string-to-string spacing.

Surface veto footprint and threshold The surface veto reduces the background from penetrating muons by identifying and rejecting high-energy air showers that reach ground level in or near it. We model its effect by splitting the projected area of the in-ice array in each zenith band into a portion that is covered by the surface veto footprint $\left(75 \mathrm{~km}^{2}\right.$ by default) and one that is not. Within the coverage of the surface veto, the background from penetrating atmospheric muons is removed entirely above a fixed threshold energy ( $100 \mathrm{TeV}$ by default), while outside it is unaffected. A $75 \mathrm{~km}^{2}$ surface array would cover the entire $240 \mathrm{~m}$ sunflower array up to zenith angles of 45 degrees, and half of the array up to 70 degrees.

Muon energy resolution $\mathrm{TeV}$ muons predominantly lose energy stochastically, leading to significant spread in the muon energy observables associated with a single true muon energy. The muon energy resolution is a parameterization of the reconstructed energy distribution after the selection described above.

Point spread function The point spread function parameterizes the distribution of the angular distance between the true muon direction and the reconstructed direction as a function of zenith angle and muon energy. The right panel of Fig. 2 shows example median opening angle distributions.

The performance criteria for cascades are simpler. The muon effective area is replaced by an effective volume of ice inside which penetrating atmospheric muons can be cleanly separated from neutrino-induced cascades. In analogy to [3], we parameterize the effective volume for the 

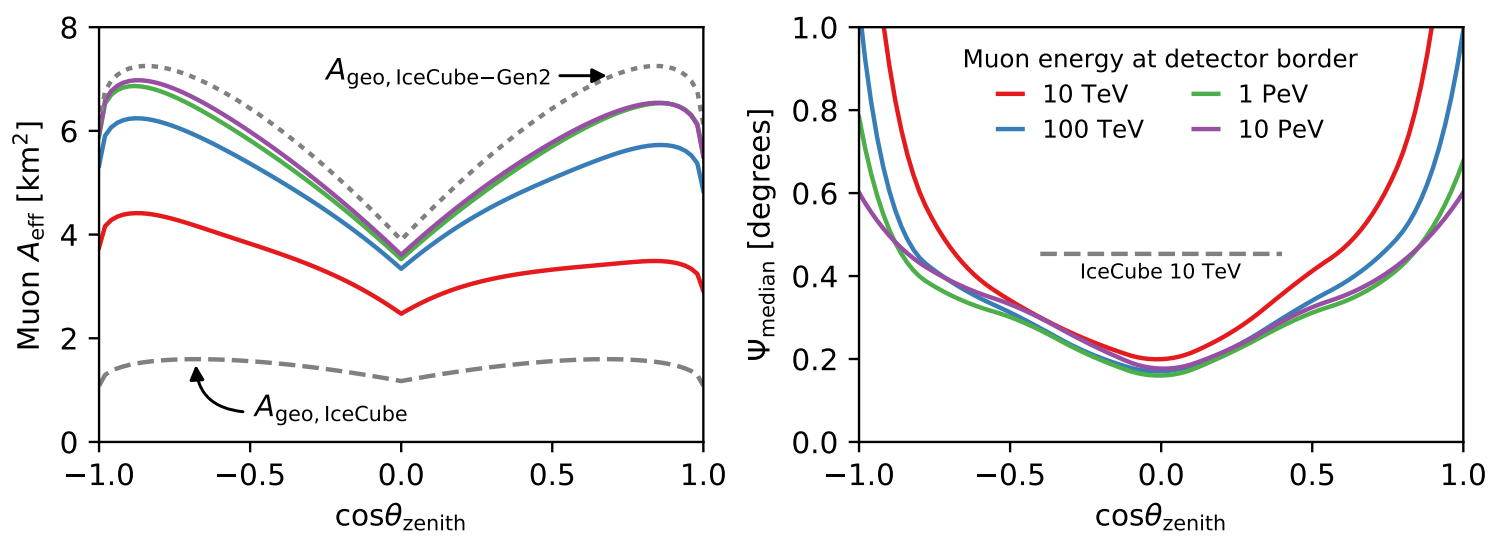

Figure 2: Muon detection and reconstruction performance of $240 \mathrm{~m}$ "sunflower" geometry. The left panel shows the average muon effective area after quality cuts as a function of zenith angle at 4 selected muon energies, evaluated at the border of the high-energy array. The geometric area of the array is shown for comparison. The right panel shows the median angular distance between the reconstructed and true muon direction. The typical angular error for IceCube is shown for comparison.

$240 \mathrm{~m}$ "sunflower" geometry as $6.3 \mathrm{~km}^{3}$ above $200 \mathrm{TeV}$ deposited energy [12] and 0 below. A cascade deposits all of its energy over a short distance, making it simpler to infer its total energy. We parametrize the deposited energy resolution as $\sigma_{\log _{10}\left(E_{\mathrm{obs}} / E_{\mathrm{dep}}\right)}=0.04 \cdot\left[1+\left(E_{\mathrm{dep}} / 1 \mathrm{PeV}\right)^{-1 / 2}\right]$. The point spread function is omitted entirely. We do not take the surface veto into account for cascade events. For simplicity's sake we also neglect the possibility that tracks would start inside the fiducial volume or that charged-current $v_{\tau}$ interactions inside the fiducial volume could be positively identified.

\subsection{Sensitivity and discovery potential}

We use these predicted event rates to calculate parameter limits for various benchmark analyses, modeling the sensitivity and discovery potential of each analysis as a binned Poisson likelihood ratio test. We define the sensitivity as the median $90 \%$ confidence level (CL) upper limit on a parameter (e.g. the flux from a candidate neutrino source) that would be obtained in repeated experiments if the parameter were 0 , and the discovery potential as the value of the parameter required to exclude 0 at a CL equivalent to $5 \sigma$ [13]. In both cases, we calculate the median test statistic using an Asimov dataset [14] rather than an ensemble of pseudo-data realizations.

Here we present two broad categories of searches: quasi-diffuse analyses, which measure the energy spectrum and flavor composition of the superposition of all astrophysical neutrino sources, and point-source analyses, which search for neutrinos from individual sources or catalogs of similar sources. The quasi-diffuse analyses use both track and cascade events, while the point-source analyses use track events only.

\subsection{Quasi-diffuse flux}

IceCube has discovered a quasi-diffuse astrophysical neutrino flux both in the starting event (cascade-dominated) [3] and up-going muon channels [5], but its origins remain unknown. The observed flux level is compatible with the widely assumed hypothesis that these neutrinos are associated with the production sites of ultra-high-energy cosmic rays (UHECR) [15]. The limited 
Figure 3: IceCube-Gen2 quasi-diffuse neutrino flux measurement after 15 years of operation. The dotted line shows a superposition of a soft $\left(E^{-2.5}\right)$ and a hard $\left(E^{-2}\right)$ component that is compatible with both the low-threshold, all-channel IceCube analysis [16] (filled grey band) and the high-threshold, muon-only analysis [5] (outlined band). The blue bars show the median range of fluxes allowed at $68 \% \mathrm{CL}$ in each range of neutrino energies, along with the number of events attributable to each range. These would allow for a clear distinction between the soft spectrum observed at lower energies (grey dashed line) and a hard spectrum that continues to the energy range of the UHECR.

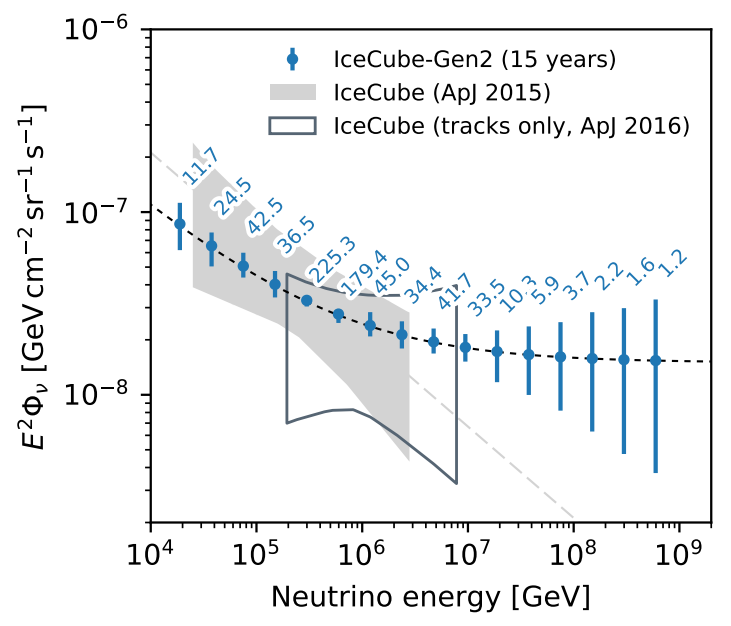

event rate in IceCube, however, prohibits strong statements about the behavior of the flux above a few PeV. To make a direct statement about the connection, we must measure the flux at higher energies.

To demonstrate the energy reach of the IceCube-Gen2 high-energy array, we construct a differential flux measurement similar to [3]. We use both the incoming track and cascade channels, and allow downgoing tracks that pass through the footprint of the surface veto. Furthermore, we assume a combined measurement using 15 years of IceCube data and 15 years of IceCube-Gen2, using muon effective areas and cascade effective volumes similar to [5] and [3] to predict the number of cascades and incoming tracks, respectively, in IceCube. As a background we assume only atmospheric neutrinos, using the fluxes of [17, 18], modified to account for the cosmic ray knee as in [5]. The signal hypothesis is a set of piecewise $E^{-2}$ power law fluxes, where each segment is allowed to vary independently. The result of such an analysis is shown in Fig. 3. The error bars on each point show the median range of fluxes that would be allowed by the data in each energy range. The flux would remain distinguishable from 0 well into the hundreds of $\mathrm{PeV}$, providing valuable overlap with radio detection, and cementing the connection between high-energy neutrinos and UHECR.

Another important aspect that can be explored with IceCube-Gen2 is the nature of the acceleration environments that produce high-energy neutrinos. If the neutrinos are produced via pion decay in the presence of strong magnetic fields, then synchrotron cooling of the pions and secondary muons will lead to breaks in the spectra of muon and electron neutrinos at the source, making the flavor ratio at Earth transition from roughly 1:1:1 to 1:1.8:1.8 over a decade in energy [19]. If the transition energy lies in the range of IceCube-Gen2, it will be observable as a break in the energy spectrum measured by IceCube-Gen2 that behaves differently in tracks and cascades.

Figure 4 shows an example of a search for flavor dependence in such a break in a case where the critical energy is $2 \mathrm{PeV}$, using the same detectors, exposures, and backgrounds as in the flux unfolding example above. Here, the model signal spectrum is divided into two components whose internal flavor compositions are allowed to vary independently. The assumed flux is $E^{2} \Phi_{v}=10^{-8} \mathrm{GeV} \mathrm{cm}^{-2} \mathrm{sr}^{-1} \mathrm{~s}^{-1}$ per flavor for $E_{v} \lesssim 1 \mathrm{PeV}$, softening to $E^{-4}$ for $E_{v} \gtrsim 10 \mathrm{PeV}$. The flavor composition measured above $1 \mathrm{PeV}$ would exclude the expected 1:1:1 at Earth at 95\% CL. 


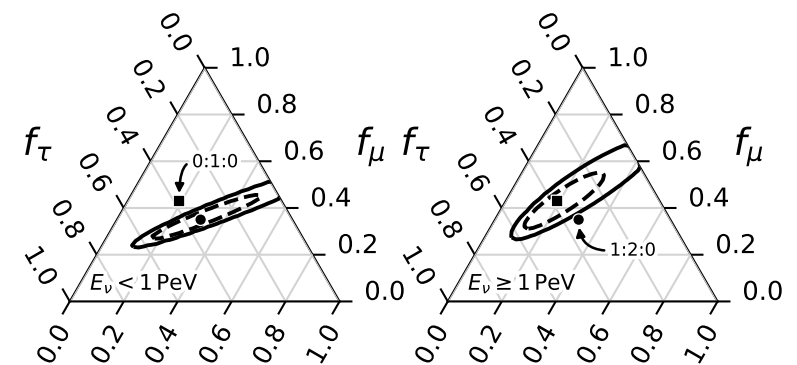

$f_{e}$

$f_{e}$

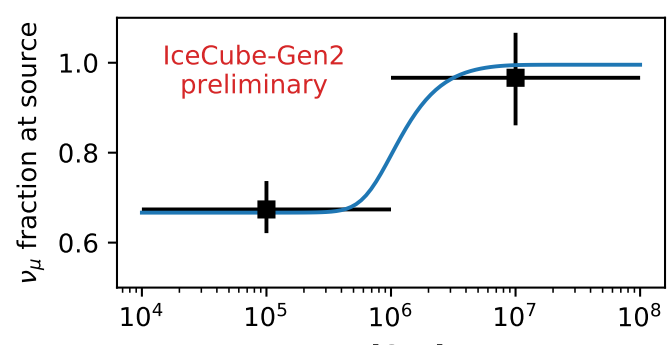

$E_{v}[\mathrm{GeV}]$

Figure 4: Measurement of a muon-damping break at $1 \mathrm{PeV}$ with IceCube-Gen2. The left panels show the constraints on the flavor ratio at Earth below and above $1 \mathrm{PeV}$, respectively. The points show the expected ratios at Earth from muon-damped pion decay $\left(\left[v_{e}: v_{\mu}: v_{\tau}\right]_{\text {source }}=0: 1: 0\right)$ and complete pion decay $(1: 2: 0)$. The dotted contours give the $68 \% \mathrm{CL}$ allowed region, while the solid lines correspond to $90 \% \mathrm{CL}$. The error bars in the right panel show the $68 \% \mathrm{CL}$ constraints on the muon-neutrino fraction at the source assuming standard oscillations over long baselines [20], while the line shows the injected flavor composition at the source.

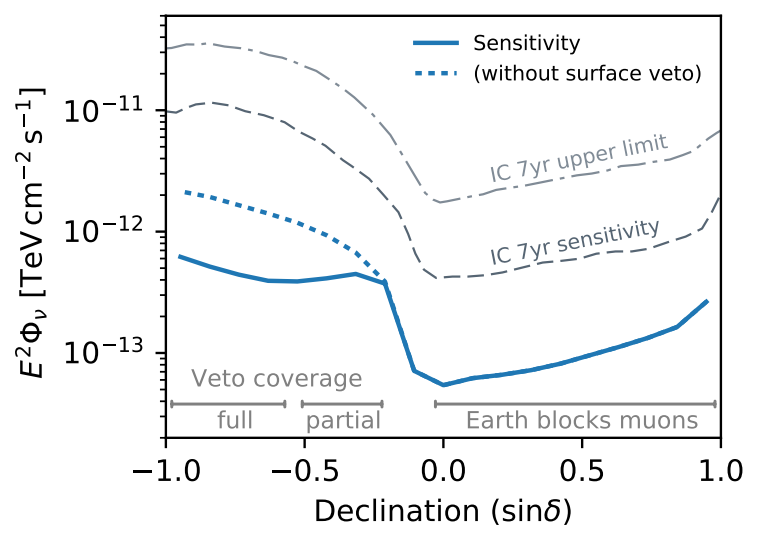

Figure 5: Integrated sensitivity for an $E^{-2}$ flux from a single source after 15 years of IceCube operation followed by 15 years of IceCube-Gen2. The sensitivity and trials-corrected upper limit of the all-sky point source search with 7 years of IceCube data [6] are shown for comparison. The discovery potential is typically 2.5 times larger than the sensitivity. The surface veto improves both discovery potential and sensitivity by a factor $\gtrsim 3$ for $\sin \delta \lesssim-0.5$.

\subsection{Point sources}

While the quasi-diffuse astrophysical neutrino flux has been observed, its sources have remained too faint to detect. The significantly larger instrumented area of IceCube-Gen2 will, however, allow sensitivity to fluxes from individual sources that are fainter than current limits.

Figure 5 shows integrated sensitivities and discovery potentials to an $E^{-2}$ flux from a single source, using only through-going track events Neutrino absorption in the Earth reduces the sensitivity towards the North Pole. In the Southern sky, overall sensitivity is reduced due to the energy threshold imposed by the surface veto and the limited target mass for neutrino interactions between the surface and the detector. Like IceCube, IceCube-Gen2's best sensitivity is at the local horizon $(\delta=0)$, where the projected density of the instrumentation and the range of neutrino-induced muons is greatest.

\section{Outlook: improved sensor designs}

The performance estimates shown above were obtained with the PINGU DOM (PDOM) [21], an updated version of the IceCube DOM that uses the same 10-inch, high-quantum-efficiency PMT 
as DeepCore. Newer sensor designs can potentially provide better sensitivity. Two new designs currently under development are the D-Egg [22, 23], with two 8-inch PMTs placed back-to-back in an ellipsoidal pressure vessel, and the mDOM [24], a smaller adaptation of the KM3NeT sensor with 24 3-inch PMTs arranged in a nearly-spherical pressure vessel. Aside from some ability to resolve the directions of photons, these differ from the PDOM primarily in the total amount and the orientation of their photon effective area, as shown in Fig. 6a. While the PDOM's single downwardfacing PMT is most sensitive to upward-going photons $(\cos \eta \sim 1)$, the D-Egg is symmetrically sensitive to upward- and downward-going photons with a total photon effective area 1.5 times greater than the PDOM, and the mDOM has nearly isotropic sensitivity with a total effective area 2.2 times greater than the PDOM. This could be increased to nearly 3 times by using super-bialkali (SBA) photocathode PMTs.

These larger sensors can improve performance either by a) increasing the total photon effective area per string, b) concentrating the same effective area in fewer modules per string, or c) allowing strings to be placed farther apart for increased neutrino effective area. Fig. $6 \mathrm{~b}$ illustrates a minimal version of the potential gains from (a) using the point source discovery potential at $\delta=0$. The improved point source sensitivity arises from an improvement in reconstruction efficiency and accuracy for muons with energies smaller than roughly $100 \mathrm{TeV}$ with increased photon effective area per sensor. While the angular resolution for horizontal muons using only the arrival time of the first photon [25] does not appear to benefit directly from the ability of segmented sensors to resolve photon directions, we expect these sensors to improve angular resolution for cascade events as well as the rejection of penetrating atmospheric muons.

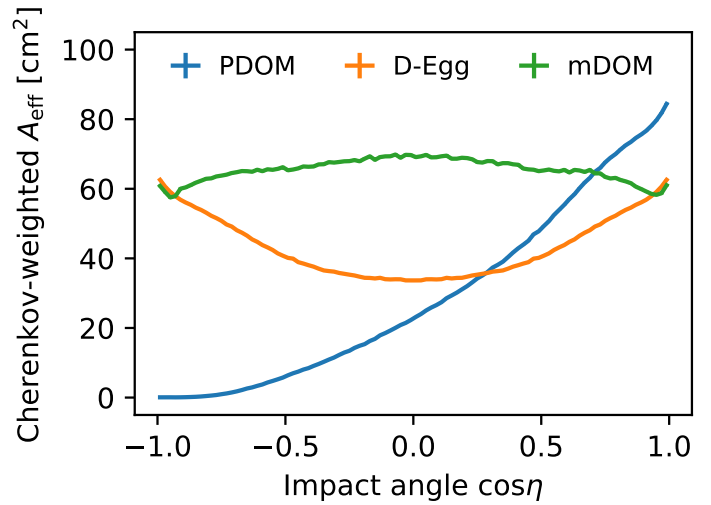

(a) Photon effective areas

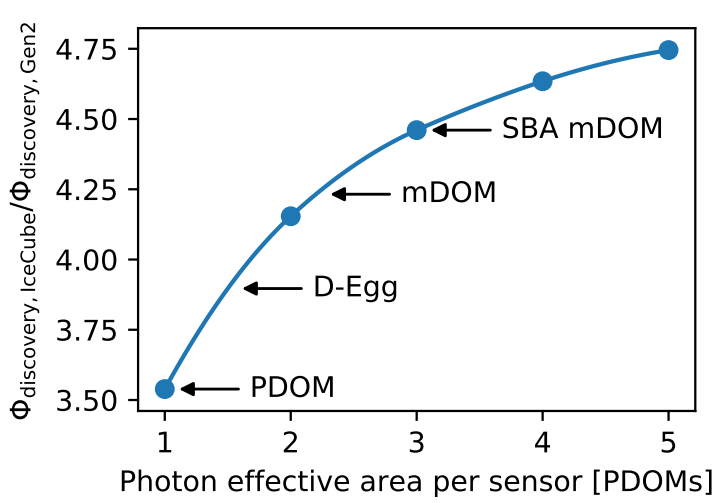

(b) Flux sensitivity vs. area per sensor

Figure 6: Sensitivity improvements from new sensor designs. (a) shows the effective area of the D-Egg and mDOM compared to the PDOM; the total area of the D-Egg is 1.5 times larger than the PDOM, while the $\mathrm{mDOM}$ is 2.2 times larger. (b) shows how larger sensors would improve the ability to discover a point source at $\delta=0$. A sunflower array with 120 strings spaced $240 \mathrm{~m}$ apart with 80 PDOMs each would be able to discover sources 3.5 times fainter than IceCube, while increasing the effective area of each sensor by 3 times (for example, by replacing each PDOM with an mDOM with SBA-photocathode PMTs) would increase this factor to 4.5 . 


\section{Summary}

IceCube-Gen2 will be a wide-band facility that detects neutrinos from several $\mathrm{GeV}$ to hundreds of PeV. Here we have presented sensitivity studies using two of its components, the high-energy in-ice array and the surface veto array. Together these can enable measurements of the astrophysical neutrino flux at the level of $E^{2} \Phi_{v}=10^{-8} \mathrm{GeV} \mathrm{cm}^{-2} \mathrm{sr}^{-1} \mathrm{~s}^{-1}$ per flavor up to hundreds of $\mathrm{PeV}$, measurements of the flavor composition of the astrophysical neutrinos in the $\mathrm{PeV}$ range, and detection of steady point sources that are 3.5 to 4.5 times fainter than those detectable with IceCube alone.

\section{References}

[1] IceCube Collaboration, M. G. Aartsen et al., JINST 12 (2017), 03 P03012.

[2] IceCube Collaboration, M. G. Aartsen et al., Science 342 (2013) 1242856.

[3] IceCube Collaboration, M. G. Aartsen et al., Phys. Rev. Lett. 113 (2014) 101101.

[4] IceCube Collaboration, M. G. Aartsen et al., Phys. Rev. Lett. 115 (2015), 8081102.

[5] IceCube Collaboration, M. G. Aartsen et al., Astrophys. J. 833 (2016), 13.

[6] IceCube Collaboration, M. G. Aartsen et al., Astrophys. J. 835 (2017), 2151.

[7] IceCube Collaboration, M. G. Aartsen et al., arXiv:1412.5106.

[8] IceCube Collaboration, PoS ( ICRC2017) 967 (these proceedings).

[9] IceCube-Gen2 Collaboration, P OS ( ICRC2017) 1055 (these proceedings).

[10] ARA Collaboration, P. Allison et al., Astropart. Phys. 70 (2015) 62-80.

[11] IceCube Collaboration, M. G. Aartsen et al., J. Phys. G44 (2017), 5054006.

[12] IceCube-Gen2 Collaboration, POS ( ICRC2017) 945 (these proceedings).

[13] G. C. Hill and K. Rawlins, Astropart. Phys. 19 (2003) 393-402.

[14] G. Cowan, K. Cranmer, E. Gross, and O. Vitells, Eur. Phys. J. C71 (2011) 1554.

[15] E. Waxman and J. N. Bahcall, Phys. Rev. D59 (1999) 023002.

[16] IceCube Collaboration, M. G. Aartsen et al., Astrophys. J. 809 (2015), 198.

[17] M. Honda, T. Kajita, K. Kasahara, S. Midorikawa, and T. Sanuki, Phys. Rev. D75 (2007) 043006.

[18] R. Enberg, M. H. Reno, and I. Sarcevic, Phys. Rev. D78 (2008) 043005.

[19] T. Kashti and E. Waxman, Phys. Rev. Lett. 95 (2005) 181101.

[20] M. C. Gonzalez-Garcia, M. Maltoni, and T. Schwetz, JHEP 11 (2014) 052.

[21] IceCube PINGU Collaboration, P. Sandstrom, AIP Conf. Proc. 1630 (2014) 180-183.

[22] IceCube-Gen2 Collaboration, POS ( ICRC2017) 1051 (these proceedings).

[23] IceCube-Gen2 Collaboration, POS ( ICRC2017) 1038 (these proceedings).

[24] IceCube-Gen2 Collaboration, POS ( ICRC2017) 1047 (these proceedings).

[25] AMANDA Collaboration, J. Ahrens et al., Nucl. Instrum. Meth. A524 (2004) 169-194. 